\title{
Seven Ventilators Challenged With Leaks During Neonatal Nasal CPAP: An Experimental Pilot Study
}

\author{
Thomas Drevhammar MD, Kjell Nilsson MD, Henrik Zetterström MD PhD, and \\ Baldvin Jonsson MD PhD
}

\begin{abstract}
BACKGROUND: Nasal CPAP is the most common respiratory support for neonates. Several factors are considered important for effective treatment, including leaks at the patient interface and the delivery of pressure-stable CPAP. Investigations of pressure stability during leaks should include both the change in the mean delivered CPAP and the pressure variation during each breath. The aim of this study was to examine the response of ventilators delivering nasal CPAP when challenged with leaks at the patient interface. METHODS: Seven ventilators providing nasal CPAP at $4 \mathrm{~cm} \mathrm{H}_{2} \mathrm{O}$ were challenged with leaks during simulated neonatal breathing. Leak was applied for 15 consecutive breaths at a constant level (1-4 L/min). RESULTS: The 2 aspects of pressure stability were evaluated by measuring the mean delivered CPAP and the amplitude of pressure swings before, during, and after leaks. The ability to maintain the delivered CPAP and the amplitude of pressure swings varied greatly among the 7 ventilators before, during, and after leaks. Four of the ventilators tested have built-in leak compensation. CONCLUSIONS: There was no simple relationship between maintaining delivered CPAP during leaks and providing CPAP with low pressure swing amplitude. Maintaining the delivered CPAP and providing this without pressure swings are 2 separate aspects of pressure stability, and investigations concerning the clinical importance of pressure stability should address both aspects. This study also shows that compensation for leaks does not necessarily provide pressure-stable CPAP. Key words: continuous positive airway pressure; intensive care; neonatal; work of breathing; ventilators; mechanical; infant newborn; equipment design. [Respir Care 2015;60(7):1000-1006. (C) 2015 Daedalus Enterprises]
\end{abstract}

\section{Introduction}

Neonatal breathing support has included noninvasive CPAP since the first publication by Gregory et al in $1971 .^{1}$ CPAP can be delivered by nasal prongs, and nasal CPAP

\footnotetext{
Drs Drevhammar and Jonsson are affiliated with the Department of Women's and Children's Health, Karolinska Institutet, Stockholm; Dr Drevhammar is also affiliated with the Department of Anaesthesiology, Östersund Hospital, Östersund; Dr Nilsson is affiliated with Ventinvent $\mathrm{AB}$, Östersund; Dr Zetterström is affiliated with the Department of Surgical Sciences, Anaesthesiology and Critical Care Medicine, Uppsala, Sweden.
}

Supplementary material related to this paper is available at http:// www.rcjournal.com.

Dr Drevhammar presented the results of this research at the Fourth Congress of the European Academy of Paediatric Societies, held October 5-9, 2012, in Istanbul, Turkey. is the recommended respiratory support in neonates. ${ }^{2}$ There are several available systems for providing nasal CPAP. Traditional systems include bubble CPAP and variable flow CPAP generators such as Infant Flow and Medijet. Bubble CPAP is generated using a constant bias flow with an expiratory resistance generated by immersion of the expiratory limb in water. Many modern ventilators are able to provide CPAP with a noninvasive patient interface such as prongs or nasal mask. It has been suggested that

\footnotetext{
This research was supported by Research and Development Unit grant 8250, Jämtland County Council, Sweden. Dr Jonsson has been engaged as a medical consultant for Maquet Critical Care. The other authors have declared no conflicts of interest.

Correspondence: Thomas Drevhammar MD, Östersund Hospital, 83183 Östersund, Sweden. E-mail: thomas.drevhammar@regionjh.se.
}

DOI: $10.4187 /$ respcare. 03718 
providing an adequate seal without leaks is an important factor when investigating nasal CPAP systems, and experimental data have shown a decrease in delivered CPAP with leaks present. ${ }^{3-5}$

Although the subject has received interest from clinicians and researchers, few in vitro investigations of nasal CPAP and leaks are available. In a static comparison of bubble CPAP and ventilator-delivered nasal CPAP, the ventilator showed less variation in mean delivered CPAP. However the method did not allow determination of dynamic variables such as imposed work of breathing, and the discussion focused entirely on bubble CPAP. ${ }^{6}$

Previously, we have used simulated neonatal breathing to investigate pressure stability. Traditional nasal CPAP systems showed an uncomplicated response to leaks: the more pressure-stable, the less reduction in delivered CPAP. ${ }^{7}$ The ventilator-driven nasal CPAP systems have a more complex design compared with traditional nasal CPAP systems. We found that some of them not only delivered CPAP but also a small pressure support, even when they were in CPAP mode. The ventilators tested also showed large variations in pressure stability. ${ }^{8}$

Large differences between in vitro performance of ventilators have been reported for noninvasive ventilation (NIV) in pediatric settings. ${ }^{9}$ If performance has an effect on outcome, investigations of respiratory support using modes such as nasal CPAP, NIV, and high frequency oscillatory ventilation may give conflicting results depending on the type of ventilator used. Hence, there is a need to investigate the capabilities of systems used for respiratory support.

A description of nasal CPAP pressure stability during leaks should include not only the change in mean delivered CPAP during leaks, but also the variation in pressure across each breath. The use of the term pressure stability may be problematic if these 2 components give conflicting results. There have been no large studies examining the response to leaks for ventilators providing nasal CPAP or describing the 2 aspects of pressure stability for this therapy. The aim of this pilot study was to examine the response of ventilators delivering nasal CPAP when challenged with leaks at the patient interface.

\section{Methods}

Research was conducted at the Karolinska Institutet (Stockholm, Sweden) and Östersund Hospital (Östersund, Sweden). Dynamic measurements during simulated neonatal breathing were performed using a mechanical lung model (ASL 5000, IngMar Medical, Pittsburgh, Pennsylvania). The setup was similar to our previous studies.7,8 The lung model reproduced a flow trace from a $3.4-\mathrm{kg}$ infant (Fig. 1) in a noncompliant mode. ${ }^{10}$ The accuracy of the mechanical lung and reproduction of flow profiles was

\section{QUICK LOOK}

\section{Current knowledge}

Noninvasive positive airway pressure is a common method of respiratory support in infants. The success of noninvasive support in this population is complicated by leaks resulting in loss of airway pressure. Mechanical ventilators vary in the ability to compensate for leaks and effectively synchronize with patient demands.

\section{What this paper contributes to our knowledge}

In a lung model, when 7 ventilators were exposed to leaks, the ability to maintain CPAP and provide low pressure swings varied greatly. The maintenance of CPAP and the pressure swings generated by breathing through the device were separate aspects of pressure stability. Some systems maintain CPAP during leaks with large pressure swings. Compensation for leaks did not necessarily provide a stable CPAP.

presented in our previous study. ${ }^{7}$ The dead space of the lung model was $210 \mathrm{~mL}$ at the start of inspiration, and compression of this volume was continuously calculated by the software. A $22 \mathrm{~mm}$ T-connector added a dead space of approximately $40 \mathrm{~mL}$ ( $10 \mathrm{~mL}$ for the test without leaks). The software did not compensate for compression of this volume.

Seven different CPAP systems with recommended tubing and humidifiers were tested (Table 1). They were combined with nasal prongs of appropriate size according to the manufacturers' guidelines. Internal diameter was used as an additional guide to find comparable prongs. Before starting the simulation, the CPAP level was adjusted to $4 \mathrm{~cm} \mathrm{H}_{2} \mathrm{O}$ on the ventilator. The Engström Carestation (GE Healthcare, Little Chalfont, United Kingdom) allowed

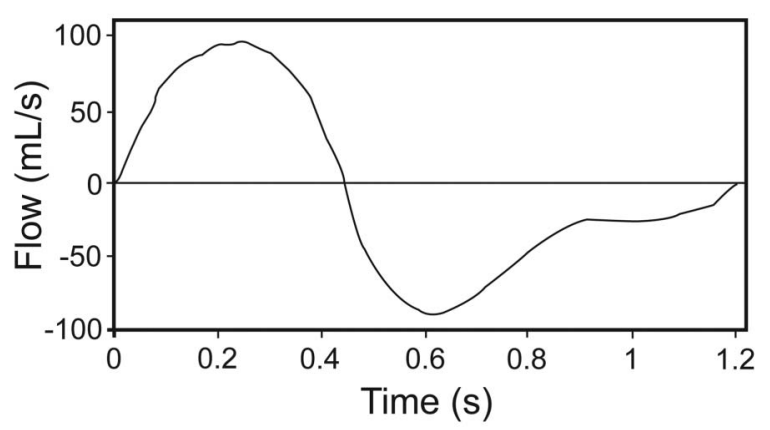

Fig. 1. The flow profiles used by the lung model from a previous study. ${ }^{7}$ Recorded flow from a healthy male newborn weighing $3.4 \mathrm{~kg}$. Breathing frequency 50 breaths $/ \mathrm{min}$, tidal volume $30.8 \mathrm{~mL}$, maximum inspiratory flow $100 \mathrm{~mL} / \mathrm{s}$, and I:E ratio 0.63. (Adapted with permission from Reference 10.) 
Table 1. The Tested Ventilators

\begin{tabular}{|c|c|c|}
\hline Ventilator & Set-up & Nasal prongs and type of system \\
\hline Avea (CareFusion, San Diego, California) & $\begin{array}{l}\text { Tubing and humidifier RT } 235 * \\
\text { Proximal pressure measure at } \\
\text { prongs. }\end{array}$ & $\begin{array}{l}\text { Hudson RCI } \dagger \text { size } 3 \text { (inner diameter } 3.5 \mathrm{~mm} \text { ). } \\
\text { Y-piece system. }\end{array}$ \\
\hline Babylog VN500 (Dräger, Lübeck, Germany) & Tubing and humidifier RT $235 .^{*}$ & $\begin{array}{l}\text { BabyFlow Prong L (inner diameter } 3.5 \mathrm{~mm} \text { ). } \\
\text { Y-piece system. }\end{array}$ \\
\hline $\begin{array}{l}\text { Engström Carestation (GE Healthcare, } \\
\text { Little Chalfont, United Kingdom) }\end{array}$ & Tubing and humidifier RT $225 . *$ & $\begin{array}{l}\text { BC4540 (inner diameter } 3.5 \mathrm{~mm} \text { ).* Y-piece } \\
\text { system with adjustable bias-flow. }\end{array}$ \\
\hline Evita XL (Dräger) & Tubing and humidifier RT $235 *$ & $\begin{array}{l}\text { Dräger size large (inner diameter } 3.5 \mathrm{~mm} \text { ). } \\
\text { Y-piece system. }\end{array}$ \\
\hline Fabian (Acutronic, Hirzel, Switzerland) & $\begin{array}{l}\text { Infant Flow nasal CPAP patient } \\
\text { circuit } \$ \text { and humidifier } \\
\text { MR290.* }\end{array}$ & $\begin{array}{l}\text { Size large (inner diameter } 3.5 \mathrm{~mm} \text { ). Variable } \\
\text { flow CPAP generator, Infant Flow. } \$\end{array}$ \\
\hline $\begin{array}{l}\text { Leoni Plus (Heinen }+ \text { Löwenstein, Bad Ems, } \\
\text { Germany) }\end{array}$ & $\begin{array}{l}\text { Humicare } 200 \text { with disposable } \\
\text { tubing. } \S\end{array}$ & $\begin{array}{l}\text { NJ1500-22 large (inner diameter } 3.3 \mathrm{~mm} \text { ). } \\
\text { Variable flow CPAP generator, NJ2000HL. }\end{array}$ \\
\hline Servo-i (Maquet, Solna, Sweden) & Tubing and humidifier RT $235 . *$ & $\begin{array}{l}\text { 1200-22 x-med (inner diameter } 3.4 \mathrm{~mm} \text { ). } \| \\
\text { Y-piece system. }\end{array}$ \\
\hline \multicolumn{3}{|c|}{$\begin{array}{l}\text { Simulations were performed with connectors and nasal interface from the producer of the tested ventilator, except where another manufacturer is indicated in footnote. } \\
\text { * Fisher \& Paykel Healthcare, Auckland, New Zealand } \\
\dagger \text { Teleflex Medical, Durham, North Carolina } \\
\text { † Viasys, Palm Springs, California } \\
\text { \& Gründler Medical, Freudenstadt, Germany } \\
\| \text { Medin Medical Innovations, Puchheim, Germany }\end{array}$} \\
\hline
\end{tabular}

adjustment of bias flow. The simulations were performed with a bias flow of $8 \mathrm{~L} / \mathrm{min}$, which, in a preliminary study, resulted in better pressure stability than the pre-set $6 \mathrm{~L} / \mathrm{min}$ (unpublished data). All tests were conducted using unheated and unhumidified air.

To evaluate the response of CPAP devices to leaks, a T-piece connector with a leak adjustment valve was interposed between the device and the lung model (Fig. 2). A constant leak flow was obtained by applying a large negative pressure over the leak adjustment valve. This leak was measured with a pneumotachometer (Fleisch No. 0; OEM Medical, Richmond, Virginia). Simulations of leaks (1, 2, 3, and $4 \mathrm{~L} / \mathrm{min}$ ) were started at breath number 30 and stopped at breath number 45 . The systems were tested once and the recordings presented graphically (Fig. 3). The mean and variance were calculated from consecutive breaths from one recording.

The recorded pressures were measured in the lung model. No pressure data were collected from the ventilator or ventilator circuit. The delivered CPAP was defined as the mean pressure for each breath and the ability to maintain CPAP as the change in this variable during leaks. The delivered CPAP before leaks was calculated for 10 consecutive breaths (number 18 to 27 ). The reduction of CPAP during leak was calculated for 10 consecutive breaths (number 33 to 42). The amplitude of pressure swings refers to the difference between lowest and highest pressure for each breath and was calculated for the same consecutive breaths as for delivered CPAP.
Recorded data were compiled using Microsoft Excel 2007 (Microsoft, Redmond, Washington) and processed in PASW Statistics 20 (IBM, Armonk, New York).

Statistical comparison was performed using an independent sample $t$ test or a one-way analysis of variance with Bonferroni corrected post hoc analysis. $P<.05$ was considered statistically significant.

\section{Results}

The delivered CPAP for the systems when subjected to leak rates of 2 and $4 \mathrm{~L} / \mathrm{min}$ is shown in Figure 3 and as supplementary data, which also includes leak rates of 1 and $3 \mathrm{~L} / \mathrm{min}$ (see Fig. 1 in the supplementary materials at http://www.rcjournal.com). A pre-set CPAP of $4 \mathrm{~cm} \mathrm{H}_{2} \mathrm{O}$ resulted in a delivered $\mathrm{CPAP}$ before leak ranging from $3.6 \mathrm{~cm} \mathrm{H}_{2} \mathrm{O}$ for the Leoni Plus (Heinen + Löwenstein, Bad Ems, Germany) to $5.0 \mathrm{~cm} \mathrm{H}_{2} \mathrm{O}$ with the Engström Carestation (Fig. 4; see also Table 1 in the supplementary materials at http://www.rcjournal.com). All comparisons were statistically significant except between Fabian and Avea.

\section{Changes in Delivered CPAP}

The response to leaks is shown in Figure 4 (complete data available in Table 2 of the supplementary materials at http://www.rcjournal.com). As the level of leak increases, the delivered CPAP decreases for most ven- 

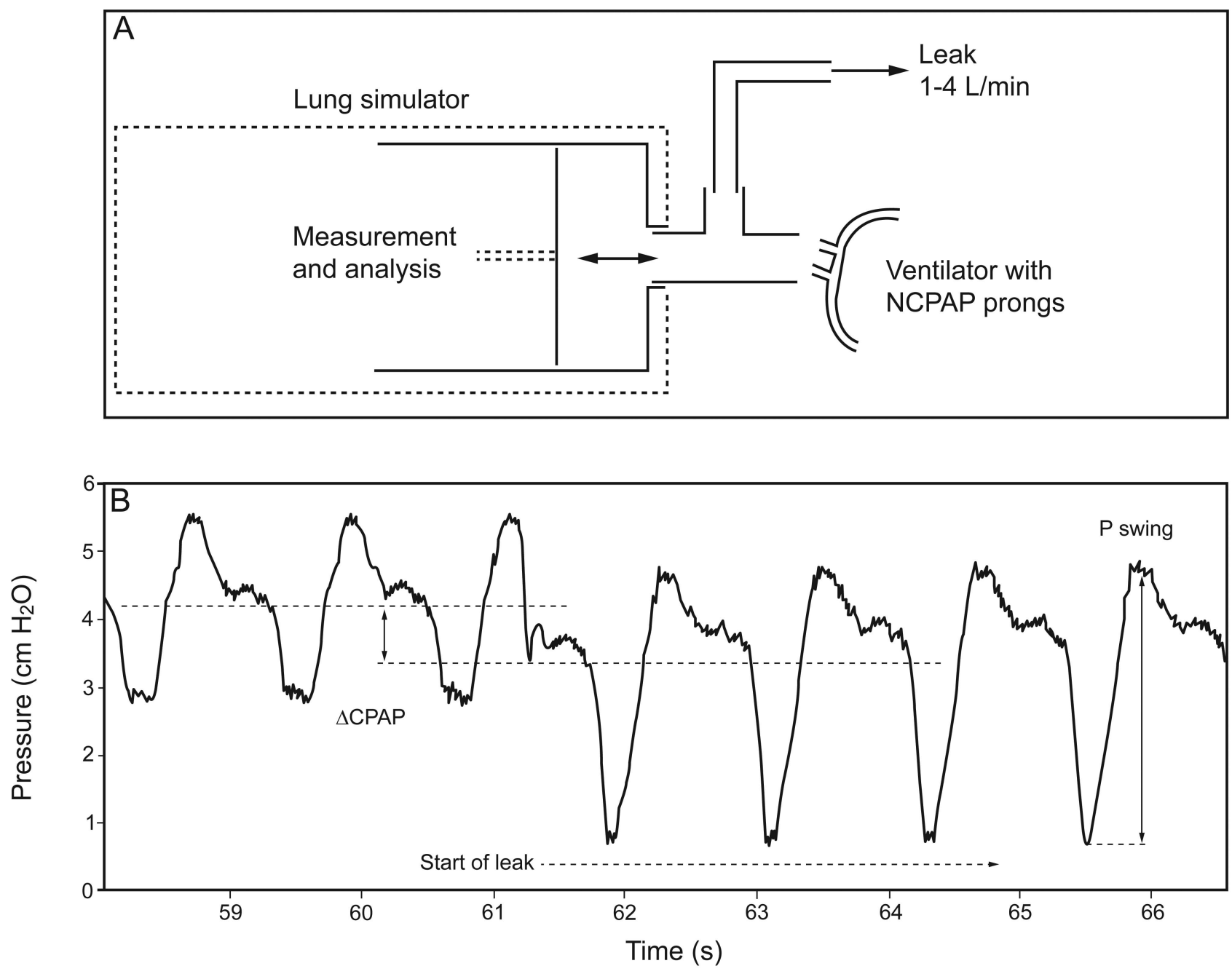

Fig. 2. Experimental setup with an example of data recorded. A: The ventilator was connected to the lung simulator with the dedicated nasal CPAP (NCPAP) system. B: A constant leak was started at breath 30 . The CPAP level and the amplitude of pressure swings (P swing) were determined before and during leak. The recording show an example of a reduction in delivered CPAP $(\triangle \mathrm{CPAP})$ and an increase in amplitude of pressure swings during leak.

tilators. Avea showed no reduction at $3 \mathrm{~L} / \mathrm{min}$. Fabian (statistically nonsignificant $2-3 \mathrm{~L} / \mathrm{min}$ ) and Servo-i (statistically nonsignificant $2-3 \mathrm{~L} / \mathrm{min})$ showed no decrease between the 2 levels of leak. The Fabian (Acutronic, Hirzel, Switzerland), Avea (CareFusion, San Diego, California), and Servo-i (Maquet, Solna, Sweden) produced the least reduction in delivered CPAP.

Four of the ventilators incorporate built-in leak compensation, resulting in a gradual compensation for the initial drop in delivered CPAP (Fig. 4). When the leak was stopped with these ventilators, a transient overshoot of the delivered CPAP was observed. The Fabian and Avea ventilators showed compensation in less than 5 breaths, whereas the Babylog VN500 (Dräger, Lübeck, Germany) and Engström Carestation ventilators did not return to the pre-leak delivered CPAP after 15 breaths.

\section{Amplitude of Pressure Swings}

The amplitude of pressure swings varied greatly among the tested ventilators, before, during, and after leak (Fig. 3; complete data available in Table 2 of the supplementary materials at http://www.rcjournal.com). The smallest amplitudes of pressure swings were recorded for the Fabian, Evita XL (Dräger), and Servo-i ventilators (both with and without leak). Pre-leak pressure swings varied between $1.4 \mathrm{~cm} \mathrm{H}_{2} \mathrm{O}$ (Fabian) and $8.5 \mathrm{~cm} \mathrm{H}_{2} \mathrm{O}$ (Leoni Plus) (Fig. 3; all comparisons were statistically significant except between Evita XL and Servo-i). The response to leak varied. An increase in amplitude of pressure swings was found in Fabian (low absolute values), Engström, and VN500. Other ventilators showed either no change or minor increase (Avea, Evita XL, Leoni Plus, and Servo-i). 

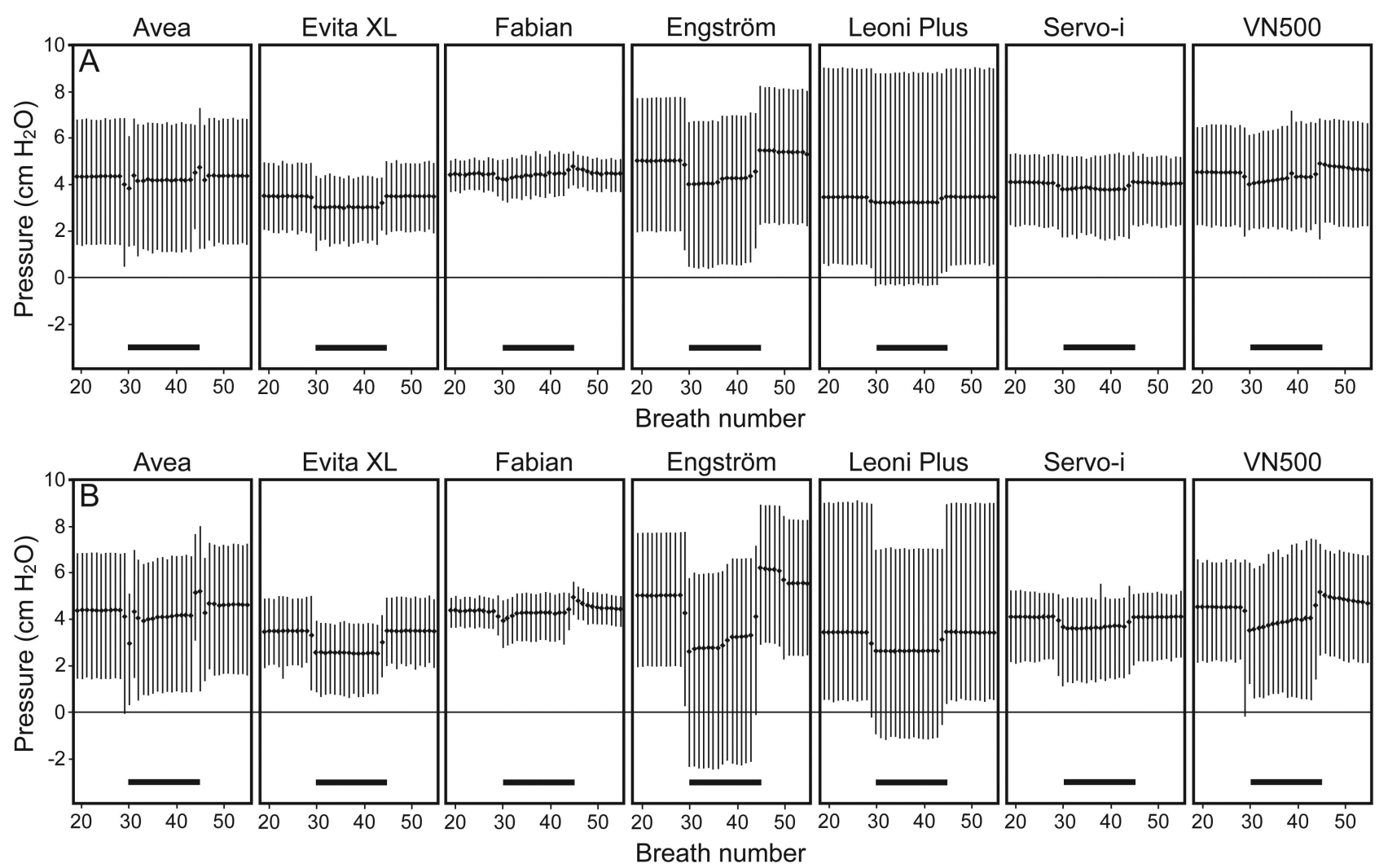

Fig. 3. Pressure response for systems during leak. Pressure change during constant leak (A: $2 \mathrm{~L} / \mathrm{min}$ leak and B: $4 \mathrm{~L} / \mathrm{min}$ leak) applied between breath number 30 and 45 (black bars) at a set CPAP of $4 \mathrm{~cm} \mathrm{H}_{2} \mathrm{O}$. Delivered mean CPAP is indicated by black points. Vertical bars indicate highest and lowest pressure for each breath, and the height of the bar represents amplitude of pressure swings for that breath.

Two ventilators (Engström and VN500) showed increased amplitude of pressure swings with increasing levels of leak (statistically significant in all comparisons of increasing leak), while others (Avea, Evita XL, Fabian, Leoni Plus, and Servo-i) showed either no change or minor increases in amplitude of pressure swings.

\section{Discussion}

The ability to maintain the pre-set CPAP level during leaks differed considerably among the ventilators (Fig. 4). Some (eg, Avea) managed to keep delivered CPAP almost unchanged. Four ventilators responded to leak with varying degrees of compensation, whereas 3 showed no sign of compensation. No ventilator allowed adjustments for leak compensation. We do not know whether leak compensation is an intended feature or just a consequence of trying to maintain adequate CPAP level.

In terms of the amplitude of pressure swings, marked differences among the systems were observed before leak was started. This has been described and discussed in our previous study. ${ }^{8}$ During leak, the amplitude of pressure swings was affected to varying degrees or virtually unchanged for some ventilators (eg, Evita XL).
Our previous study of traditional CPAP and leak showed that systems with a lower amplitude of pressure swings also showed less reduction in delivered CPAP when exposed to leaks. ${ }^{7}$ This uncomplicated relationship could not be seen in the tested ventilators of this study. There are ventilators that maintain delivered CPAP during leaks but do so with large pressure swings (eg, Engström Carestation). Other systems, such as Evita XL, showed lower amplitude of pressure swings but a larger reduction in delivered CPAP during leaks. When combining the results, we could not identify a clear pattern. This was an unexpected finding and will make future research on nasal CPAP delivered by ventilators complex. The explanation for the differences in performance are most likely related to interactions between flow and pressure sensors, valves, tubing, interface, and algorithms.

\section{Limitations}

The main limitation with in vitro studies is the obvious difference between the test conditions and the clinical setting. The ventilators in this study were challenged by constant leak. In clinical use, the leak would be variable and depend on several factors, such as mouth opening and the 

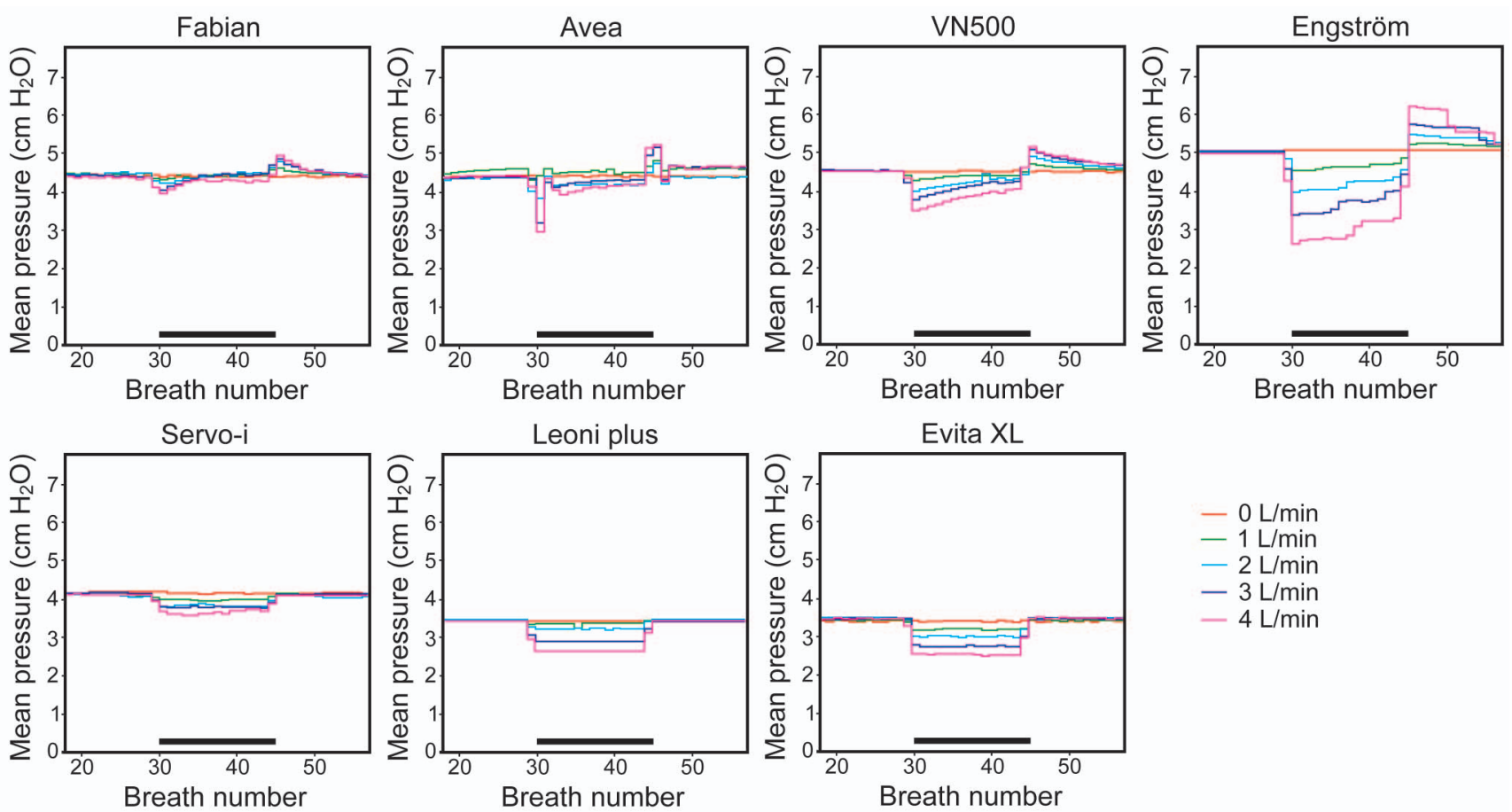

Fig. 4. Delivered mean CPAP change during leak. Delivered mean CPAP change during constant leak (1-4 L/min) applied between breath number 30 and 45 (black bars) at a set CPAP of $4 \mathrm{~cm} \mathrm{H}_{2} \mathrm{O}$. Simulations without leak $(0 \mathrm{~L} / \mathrm{min})$ were included as baseline reference. The ventilators in the top row compensate for leak.

seal at the nasal interface. Using a variable leak would have made comparisons among systems difficult, because the leak would not be comparable and influenced by both the CPAP level and the pressure stability of the system. In a clinical setting, there is a probable interaction among pressure stability, CPAP level, and a variable leak. The latter will certainly increase during expiration and be aggravated by unstable CPAP. Our results should not be used for clinical recommendations, since neither leak nor pressure stability has proven to be clinically important. This uncertainty does not imply that the uneven performance is unimportant but should warrant further clinical research on the influence of ventilator performance.

The ventilators were tested with one breath profile and at one CPAP level. The response to other patterns of breathing may be different, and the small pressure support delivered by some ventilators may give asynchronies under some conditions. The CPAP level of $4 \mathrm{~cm} \mathrm{H}_{2} \mathrm{O}$ was chosen to allow comparison with our previous study of conventional CPAP systems. ${ }^{7}$

The pressure fluctuation during one simulated breath was examined by the amplitude of pressure swings. This variable was used, as it is less complex than other measurements such as the pressure-time product or imposed work of breathing. The low level of pressure support provided by some ventilators makes it very difficult to calculate measures such as imposed work of breathing. De- tailed investigation of these systems requires methods for detection and quantifying pressure support, which were unavailable.

The measured variables in this study have low variance. This was expected, as mechanical lung models allow stable measuring conditions. The low variance means that comparisons of tests are often statistically significant, even at levels that are clinically unimportant.

\section{Clinical and Research Implications}

In contrast to our previous investigations of traditional CPAP systems, this study showed that maintaining delivered CPAP and minimizing pressure swings are 2 different and unlinked aspects of pressure stability. ${ }^{7}$ Future studies should investigate and consider them separately.

The ventilators that provided leak compensation showed mixed results for the reduction in CPAP during leak and amplitude of pressure swings. We conclude that the phrase leak compensation is no guarantee for a more pressurestable system.

The clinical importance of maintaining delivered CPAP and avoiding excessive pressure swings is poorly investigated. However, the pronounced disparities between different systems revealed in this study might be important. The physiological response and complications during CPAP treatment probably correlate to pressure changes at 
different parts of a breath. This could generate several research questions: Are high peak pressures correlated with pneumothorax or barotrauma? Is a large pressure decrease during inspiration associated with collapse and atelectrauma (repeated opening and closing of lung units)? Is the overall imposed work of breathing correlated with failure on nasal CPAP?

If pressure stability is essential, generalizing results from a study using one particular ventilator might not be appropriate. Furthermore, the use of CPAP systems with different pressure stability characteristics in clinical trials implies a risk of attenuating the clinical effect. Findings such as ours point to the need to take CPAP system performance into account when designing and interpreting clinical studies. The large differences in performance among ventilators have also been investigated in other modes such as pediatric NIV. ${ }^{9}$ There are several clinical studies that do not discuss the effect that uneven performance might have. For the neonatal population, this includes large pragmatic studies using nasal CPAP treatment in the control group. ${ }^{11,12}$

\section{Conclusions}

When exposed to leaks, the ability to maintain mean CPAP and provide low amplitude of pressure swings varied greatly among the 7 ventilators. The study shows that during leak the 2 aspects of pressure stability can give conflicting results. The maintenance of mean CPAP and the pressure swings generated by breathing through the device are separate aspects of pressure stability. There are systems that maintain mean CPAP during leak but do so with large pressure swings. This study also shows that compensation for leak does not necessarily give pressurestable CPAP.

This study as well as our previous research has shown large differences in performance of CPAP systems. The results should be used as an initial basis for interpreting performance and outcome of nasal CPAP systems and not as a guide for making clinical decisions. They should also encourage researchers to reflect on the performance of the systems used in clinical trials and discuss the impact this could have on outcome.

\section{ACKNOWLEDGMENTS}

James Bembridge MSc provided excellent help through review of the language and discussion of concepts and terminology.

\section{REFERENCES}

1. Gregory GA, Kitterman JA, Phibbs RH, Tooley WH, Hamilton WK. Treatment of the idiopathic respiratory-distress syndrome with continuous positive airway pressure. N Engl J Med 1971;284(24): 1333-1340.

2. Sweet DG, Carnielli V, Greisen G, Hallman M, Ozek E, Plavka R, et al. European consensus guidelines on the management of neonatal respiratory distress syndrome in preterm infants-2013 update. Neonatology 2013;103(4):353-368.

3. Morley C. Which neonatal nasal CPAP device should we use in babies with transient tachypnea of the newborn? J Pediatr (Rio J) 2011;87(6):466-468.

4. De Paoli AG, Lau R, Davis PG, Morley CJ. Pharyngeal pressure in preterm infants receiving nasal continuous positive airway pressure. Arch Dis Child Fetal Neonatal Ed 2005;90(1):F79-F81.

5. Chilton HW, Brooks JG. Pharyngeal pressures in nasal CPAP. J Pediatr 1979;94(5):808-810.

6. Kahn DJ, Courtney SE, Steele AM, Habib RH. Unpredictability of delivered bubble nasal continuous positive airway pressure: role of bias flow magnitude and nares-prong air leaks. Pediatr Res 2007; 62(3):343-347.

7. Drevhammar T, Nilsson K, Zetterström H, Jonsson B. Comparison of seven infant continuous positive airway pressure systems using simulated neonatal breathing. Pediatr Crit Care Med 2012;13(2): e113-e119.

8. Drevhammar T, Nilsson K, Zetterström H, Jonsson B. Comparison of nasal continuous positive airway pressure delivered by seven ventilators using simulated neonatal breathing. Pediatr Crit Care Med 2013;14(4):e196-e201.

9. Oto J, Chenelle CT, Marchese AD, Kacmarek RM. A comparison of leak compensation during pediatric noninvasive ventilation: a lung model study. Respir Care 2014;59(2):241-251.

10. Moa G, Nilsson K, Zetterström H, Jonsson LO. A new device for administration of nasal continuous positive airway pressure in the newborn: an experimental study. Crit Care Med 1988;16(12):12381242.

11. Kirpalani H, Millar D, Lemyre B, Yoder BA, Chiu A, Roberts RS A trial comparing noninvasive ventilation strategies in preterm infants. N Engl J Med 2013;369(7):611-620.

12. Manley BJ, Owen LS, Doyle LW, Andersen CC, Cartwright DW, Pritchard MA, et al. High-flow nasal cannulae in very preterm infants after extubation. N Engl J Med 2013;369(15):1425-1433. 